Moscow, Russia; Vascular Rheumatology, Moscow, Russian Federation; ${ }^{2}$ Russian Medical Academy of Continuing Professional Education, Ministry of Health of Russia, Department of Rheumatology, Moscow, Russian Federation; ${ }^{3}$ V.A. Nasonova Research Institute of Rheumatology, Moscow, Russia; Department of Rheumatology, Moscow, Russian Federation; ${ }^{4}$ V.A. Nasonova Research Institute of Rheumatology, Moscow, Russia;, Laboratory of Medical and Social Problems of Rheumatology, Moscow, Russian Federation

Background: Classification criteria for SLE need validation and selection of the most advanced ones for clinical practice

Objectives: To validate Systemic Lupus International Collaborating Clinics (SLICC)-12, American College of Rheumatology (ACR)-97 and 2019 European League against Rheumatism/American College of Rheumatology (EULAR/ACR2019) for Systemic

Lupus Erythematosus classification criteria on a patient cohort from V.A. Nasonova Scientific Research Institute of Rheumatology (Moscow)

Methods: This prospective study included 252 ( $n=152$ with SLE and $n=100$ non SLE: 74 - with systemic sclerosis (SC) and 26 - with primary antiphospholipid syndrome (pAPS)) patients who were sequentially admitted to the 4th Rheumatology Department from December 2018 to June 2020. All of the patient records were reevaluated by expert rheumatologist in order to determine if they agree with the diagnosis. For every patient, a check-list of SLE-related features was filled out. The association between clinical diagnosis and diagnosis generated on the basis of ACR-97, SLICC-12 and EULAR/ACR-2019classification criteria was assessed. The overall sensitivity and specificity of ACR-97, SLICC-12 and EULAR/ACR-2019 classifications, as well as the sensitivity and specificity according to disease duration was calculated. The predictive value of every criterion in ACR-97, SLICC-12 and 2019 EULAR/ACR classification was assessed using logistic regression analysis and receiver operating characteristic (ROC) curves.

Results: According to ACR-97 criteria, reliable SLE was diagnosed in 131 (86\%), according to SLICC-2012 - in 145 (95.3\%) and according to EULAR/ACR-2019 - in $144(94.7 \%)$ of 152 patients, respectively. We identified the criteria that were significantly more represented in the SLE-group. The sensitivity and specificity (Table 1) did not change depending on the ANA titers (1/160 and 1/320) and the duration of the disease.

Table 1. The sensitivity and specificity of SLE criteria in 152 SLE patients and 100 non SLE patients

\begin{tabular}{lcccc}
\hline SLE classification criteria & AUC, 95\% Cl & Cut point & Sensivity & Specifity \\
\hline ACR-97 & $0.654(0.550-0.758)$ & 4 & 89.7 & 36 \\
SLICC-12 & $0.616(0.505-0.728)$ & 4 & 93.1 & 43 \\
2019 EULAR/ACR & $0.609(0.492-0.727)$ & $10^{*}$ & 97.4 & 48
\end{tabular}

Note. ACR-97 - American College of Rheumatology classification from 1997: SIICC-12 Systemic lupus International Collaboration Clinics classification from 2012; 2019 European League Against Rheumatism/American College of Rheumatology. ANA - anti-nuclear antibody more than 1/320 $n=116$; ANA $<320 n=36$. AUC - area under the ROC curve; Cut point - reference point for the number of criteria, ${ }^{*}$ number of points $\geq 10$.

When excluding patients with SC in a single-factor logistic model of patients with SLE and pAPS, indicating the number of criteria, the sensitivity was for ACR-97 - 86, for SIICC-12 - 95, for 2019 EULAR/ACR - 95, the specificity was 100, 62 and 62 , respectively.

Conclusion: Evaluation of the criteria by level ANA revealed the highest sensitivity for 2019 EULAR/ACR and SLICC-12, the specificity was low for all three criteria. In the analysis of patients with SLE and pAPS, the sensitivity was highest for 2019 EULAR/ACR and SLICC-12, the specificity was 100 for ACR-97.

Disclosure of Interests: None declared

DOI: 10.1136/annrheumdis-2021-eular. 1051

\section{AB0304 LEVELS AND TRENDS IN PREMATURE MORTALITY BURDEN DUE TO SYSTEMIC LUPUS ERYTHEMATOSUS IN CUBA}

H. Hernández-Negrín ${ }^{1}{ }^{1}$ Universidad de Ciencias Médicas de Villa Clara Internal Medicine, Santa Clara, Cuba

Background: Analysis of the cause of premature mortality is an essential function of public health surveillance. A variety of methods have been used to accurately assess and report the level and trends of premature mortality; however, many have significant limitations, particularly in capturing actual early deaths. Years of life lost (YLL) has been recognized as a robust and comprehensive measure of premature mortality. ${ }^{1}$ Systemic lupus erythematosus (SLE) is among the leading causes of premature mortality burden in young women in the United States and premature mortality from SLE is increasing in Latin America. ${ }^{2,3} \mathrm{How}-$ ever, the premature mortality burden from SLE has not been fully characterized in Cuba.
Objectives: To identify levels and trends in premature mortality burden due to SLE in Cuba during 2001-2018.

Methods: Data were obtained from the mortality database of the Ministry of Public Health of Cuba (International Classification of Diseases-10, code 32 as underlying cause of death). YLL were calculated using the standard life expectancy of the WHO's global health estimates. Age- and sex-specific YLL rates were calculated using the Cuban mid-year population estimates and the age-standardized YLL rate (ASYR) was estimated by the direct method using the WHO's standard population. The 2001-2018 temporal trends were analyzed using Jointpoint software.

Results: During 2001-2018, 1475 patients died from SLE. These deaths contributed 66605 YLL (women= 59 166; men=7 433), with a mean of 45.1 YLL per death (women= 45.2; men=44.5). The 40-44 age group was the most affected in both sexes with 9266 YLL (women=8 177; men=1 086). The ASYR was higher in 2018 than in 2001 for both sexes (Figure 1). A significantly increasing trend in ASYR due to SLE was identified throughout the period (average annual percent change $[\mathrm{AAPC}]=1.9 ; 95 \%$ confidence interval $[\mathrm{Cl}]=0.9$ to 3.0 ), more pronounced in men $(A A P C=7.0 ; 95 \% \mathrm{Cl}=3.5$ to 10.6) than in women $(\mathrm{AAPC}=1.3$ $95 \% \mathrm{Cl}=0.3$ to 2.3 ).

Conclusion: The high levels and growing trends in premature mortality burden from SLE in Cuba, demands its recognition as an important health problem and immediate actions that help mitigate it.

\section{REFERENCES:}

[1] Martinez R, Soliz P, Caixeta R, Ordunez P. Reflection on modern methods: years of life lost due to premature mortality-a versatile and comprehensive measure for monitoring non-communicable disease mortality. Int $\mathrm{J}$ Epidemiol. 2019; 48(4): 1367-1376.

[2] Yen EY, Singh RR. Lupus - an unrecognized leading cause of death in young women: Population-based study using nationwide death certificates, 2000 2015. Arthritis Rheumatol. 2018;70:1251-5.

[3] Soares CR, Viana K, Moraes-Santos F, Vieira CL, Lamarão F, Iglesias-Rodriguez $M$. Mortality rate and premature mortality due to systemic erythematosus lupus in Latin America US and England and Wales. Value Health. 2015;18:A805-81.

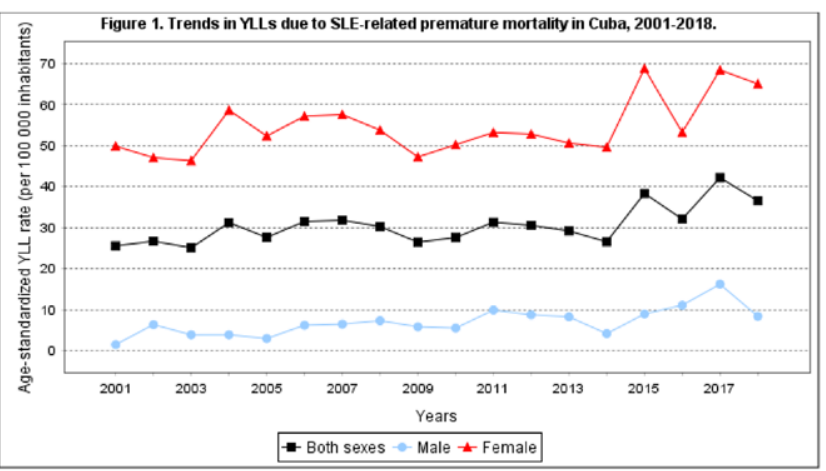

Disclosure of Interests: None declared

DOI: 10.1136/annrheumdis-2021-eular.1147

\section{$\mathrm{AB} 0305$}

IS THERE HYPERCOAGULATION IN PATIENTS WITH ANTIPHOSPHOLIPID SYNDROME AND BEHCET'S DISEASE?

N. Seredavkina ${ }^{1}$, T. Reshetnyak ${ }^{1,2}$, T. Lisitsyna ${ }^{1}$, A. Lila ${ }^{1,2} .{ }^{1}$ VA Nasonova Research Institute of Rheumatology, Department of Vascular Rheumatology, Moscow, Russian Federation; ${ }^{2}$ Federal State Budgetary Educational Institution of Further Professional Education "Russian Medical Academy of Continuous Professional Education" of the Ministry of Healthcare of the Russian Federation, Department of Rheumatology, Moscow, Russian Federation

Background: Whereas antiphospholipid syndrome (APS) is a non-inflammatory vasculopathy and is associated with thrombosis in $98 \%$ of cases, Behcet's disease (BD) is a systemic vasculitis of unknown etiology, characterized by vascular damage of any calibre. Both venous and arterial thromboses occur in $45 \%$ of BD patients and are associated not with hypercoagulable disease but with inflammatory changes in the vascular wall mediated by hypersecretion of pro-inflammatory cytokines and endothelial cells dysfunction. Thrombodynamics (TD) is a new global test for diagnosing plasma haemostasis disorders, identifying bleeding and thrombosis risks, and can be used to detect a prothrombotic state and assess the influence of disease activity and course on the hypercoagulation process.

Objectives: comparative assessment of TD in patients with APS and BD before anticoagulant therapy (AC)

Methods: The study included 20 patients ( 9 APS and $11 \mathrm{BD}$ ) and 8 age and sexmatched healthy controls $(\mathrm{HC})$. None of the subjects received AC. Thromboses 
in past history were registered in 5/9 (55\%) APS patients and 2/11 (18\%) BD patients and none of HC. Fetal loss occurred only in women with APS (4/4 $(100 \%)$ who had pregnancy during the disease). All the patients were hospitalized and underwent fool investigation according to the diagnosis including TD, local coagulation tests and antiphospholipid antibodies profile.

Results: the velocity of clot growth in APS was lower, than in BD and in HC: 23.7 [22.6; 24.7] vs 29.0 [28.2; 34.4] and 31.1 [28.9; 33.5] Um/min, respectively $(\mathrm{p}=0.001)$. Clot size at 30 minutes in APS also was lower, than in BD and HC: 972.1 [921.3; 1007.4] vs 1152.7 [1098.3; 1225.4] and 1226.6 [1140.5; 1295.1] Um, respectively $(p=0.001)$. Spontaneous clotting was registered only in 2 BD patients in mean time 2 minutes. Clot density and lag time (Tlag, the delay between the test start and the onset of clot formation) were the same in all three groups. Prolonged APTT was found in APS $(33.7[30.6 ; 47.1] \mathrm{sec})$ and normal APTT in BD $(30.9$ [29.1; 31.1] sec) and HC (29.7 [28.2; 30.8] sec). Increased soluble fibrin-monomer complexes were revealed in all APS patients (100\%), 91\% BD patients and $25 \% \mathrm{HC}(\mathrm{p}=0.01)$. After interpretation the TD results were distributed as follows: hypocoagulation was noted in 1 APS patient with a positive lupus anticoagulant, while all other APS patients had normocoagulation. Thrombotic readiness status (TRS) was diagnosed only in $2 \mathrm{BD}$ patients. The frequency of normocoagulation and hypercoagulation did not differ between BD and HC. Local coagulation tests (APTT, thrombin time, prothrombin time) were the same depending on hypo- and hypercoagulation by TD results. Fibrinogen level in BD patients with hypercoagulation was higher, than in BD patients with normocoagulation and TRS: $4.2[3.6 ; 4.7]$ vs $2.8[2.7 ; 3.0]$ and $2.8[2.7 ; 2.8] \mathrm{g} / \mathrm{l}$ respectively, $\mathrm{p}=0.04$. BD activity by Behcet's Disease Current Activity Form correlated with stationary velocity of clot growth $\left(R_{s}=0.68, p<0.05\right)$.

Conclusion: Thrombodynamics results showed: before anticoagulant therapy there were normocoagulation with a tendency to hypocoagulation in APS and hypercoagulation in BD. In BD hypercoagulation associated with disease activity. Disclosure of Interests: None declared

DOI: 10.1136/annrheumdis-2021-eular.1319

\section{AB0306 OBESITY PHENOTYPES IN SYSTEMIC LUPUS ERYTHEMATOSUS PATIENTS}

L. Kondrateva ${ }^{1}$, T. Panafidina ${ }^{1}$, T. Popkova ${ }^{1}$, M. Cherkasova ${ }^{2}$, A. Lila ${ }^{3}$, E. Nasonov ${ }^{3}{ }^{7}$ V.A.Nasonova Research Institute of Rheumatology, Department of Systemic Rheumatic Diseases, Moscow, Russian Federation; ${ }^{2}$ V.A. Nasonova Research Institute of Rheumatology, Immunology and Molecular Biology of Rheumatic Diseases, Moscow, Russian Federation; ${ }^{3}$ V.A.Nasonova Research Institute of Rheumatology, Scientific Department, Moscow, Russian Federation

Background: The so-called "obesity paradox" when cardiovascular risk is lower in overweight compared to lean patients accompanies some chronic conditions. A possible explanation for this phenomenon comes from variability of obesity phenotypes, i.e., "classical" obesity as exemplary perception of obesity is equal to increased body mass index (BMI) and metabolic disorders; but there are also "metabolically healthy" overweight individuals (increased BMI without metabolic disorders), and "latent" obesity phenomenon (when normal weight is associated with metabolic disorders, especially with insulin resistance (IR) and adipocytokines imbalance). Serum leptin concentrations increase has been established in obese, therefore, this adipocytokine synthesized in adipose tissue, can be used as a marker of body fat mass. Higher cardiovascular risks are known even in young patients with systemic lupus erythematosus (SLE), but obesity phenotypes have not been studied.

Objectives: To find out the rate of various obesity phenotypes and to identify factors contributing to "latent" obesity in SLE pts without diabetes mellitus (DM) or hyperglycemia.

Methods: A total of 49 SLE pts (46 women, 3 men, 40 [33;48] years old) without established DM or hyperglycemia were enrolled in the study. The median disease duration was $3,0[0,7 ; 8,0]$ years, SLEDAI-2K was $5[2 ; 8]$. SLE pts were treated with glucocorticoids (GC) (84\%) and hydroxychloroquine (78\%), immunosuppressive drugs (20\%) and biological agents (10\%). Insulin levels were measured using electrochemiluminescence assay Elecsys (Roche Diagnostics), serum leptin concentrations were estimated using ELISA (DBS-Diagnostics Biochem Canada Inc.). IR was defined as Homeostasis Model Assessment of Insulin Resistance index (HOMA-IR) $\geq 2,77$. Leptin levels were considered elevated at values $\geq 11,1 \mathrm{ng} / \mathrm{ml}$ for women, $\geq 5.6 \mathrm{ng} / \mathrm{ml}$ for men. The overweight / obesity status was determined by World Health Organization criteria in patients with body mass index $(\mathrm{BMI}) \geq 25 \mathrm{~kg} / \mathrm{m}^{2}$.

Results: Overweight / obesity were established in $45 \%$ of pts, normal BMI was in $55 \%$ of SLE pts. The combination of IR and high leptin levels was found in $32 \%$ of overweight / obese pts and $11 \%$ of pts with normal BMI $(p=0,2)$, an isolated increased leptin levels - in $64 \%$ and $41 \%$, respectively $(p=0,1)$. Metabolic disturbances were absent in $4 \%$ of overweight pts and $48 \%$ of pts with a normal BMI $(p=0,001)$. Thus, "classical" obesity was found in $43 \%$ of cases, "metabolically healthy" obesity - in $2 \%$, and "latent" obesity - in $29 \%$ of SLE pts. Leptin levels correlated with BMI $(r=0,5, p=0,02)$, waist circumference $(r=0,5$, $p=0,02)$ in the overweight / obesity group, and with disease duration $(r=0,5$ $p=0,02)$, SLEDAI-2K ( $r=-0,6, p=0,002)$, anti-dsDNA ( $r=-0,7, p<0,001)$, C3 complement $(r=0,5, p=0,01)$, maximum $(r=0,7, p<0,001)$ and current $\mathrm{GCs}$ doses $(r=0,4, p=0,03)$ in patients with normal weight. There was a trend to association between leptin levels and duration of GCs therapy in SLE pts with normal BMI $(\mathrm{r}=0,4, p=0,06)$.

Conclusion: Metabolic disorders - most often increased leptin levels - were diagnosed in the majority of overweight / obese patients, as well as in about $50 \%$ of SLE patients with normal weight. There were only isolated cases of "metabolically healthy" obesity in SLE patients. Metabolically obese normal weight ("latent" obesity) phenotype was strongly associated with GCs therapy and decreased disease activity.

Disclosure of Interests: None declared

DOI: 10.1136/annrheumdis-2021-eular.1356

\section{\begin{tabular}{|l|l}
\hline AB0307 & IS THERE ANY INFLUENCE OF THE ANTIRHEUMATIC
\end{tabular} THERAPY ON ECHOCARDIOGRAPHIC FINDINGS IN PATIENTS WITH SYSTEMIC LUPUS ERYTHEMATOSUS?}

T. Panafidina ${ }^{1}$, T. Popkova ${ }^{1}$, L. Kondrateva ${ }^{1}$, A. Volkov ${ }^{2}$, E. Nasonov ${ }^{3}$, A. Lila ${ }^{3}$. ${ }^{1}$ V.A.Nasonova Research Institute of Rheumatology, Department of Systemic Rheumatic Diseases, Moscow, Russian Federation; ${ }^{2}$ V.A. Nasonova Research Institute of Rheumatology, Department of Instrumental Diagnostics, Moscow, Russian Federation; ${ }^{3}$ V.A.Nasonova Research Institute of Rheumatology, Scientific Department, Moscow, Russian Federation

Background: Cardiovascular diseases are becoming the leading cause of death among SLE patients due to increasing life-spans. Transthoracic echocardiography (TTE) is a routine and widely available modality in everyday clinical practice useful to identify specific pathological cardiac changes and predictors of heart failure.

Objectives: The goal was to identify potential abnormalities in the TTE findings in SLE patients, with and without antirheumatic therapy.

Methods: This is a prospective cross-sectional study including 91 pts $(91 \%$ females, aged 32[28-41]years (median [interquartile range 25\%-75\%]) with SLE (SLICC 2012 criteria). All patients were divided into 2 groups: the 1st group was composed of "untreated" patients and the 2 nd - of patients receiving antirheumatic therapy. The 1st group included 43pts (93\% females) aged 31[27-40]years who were not receiving steroids, immunosuppressants and biological agents at the time of enrollment, $5(12 \%)$ of them were on hydroxychloroquine $(\mathrm{HCQ}$ ) therapy $200 \mathrm{mg} /$ day. The $2 \mathrm{nd}$ group is represented by $48 \mathrm{pts}$ ( $89 \%$ females) with median age $34[28-45]$ years. Out of them $47(98 \%)$ patients were on prednisone therapy at $10[8-15] \mathrm{mg} /$ day, $10(21 \%)$ - on cyclophosphamide, $6(13 \%)$-azathioprine, $4(8 \%)$-mycophenolate mofetil, $4(8 \%)$-methotrexate, $37(71 \%)-\mathrm{HCQ}$, and $9(19 \%)-$ on biologic (rituximab, belimumab). Both groups were matched by age and gender. Patients receiving antirheumatic therapy (group 2) had longer disease duration (96 vs 18 months, $p<0,00001$ ), lower disease activity (SLEDAI-2K 4 vs 11 scores, $p<0,001$ ), higher SLICC/DI ( 1 vs 0 score, $p<0,001$ ); lower percentage of them had skin lesions ( 11 vs $57 \%$, p<0,0001), arthritis (22 vs $52 \%$, $\mathrm{p}<0,05)$ and hematological disorders (24 vs $74 \%, p<0,0001)$ than "untreated" patients from the 1st group.

Results: Valve insufficiency with varying degree of clinically insignificant regurgitation and pericarditis were the commonest pathology found in "untreated" and "treated" SLE patients based on TTE data. No differences in rates of valve insufficiency $(95 \%$ and $83 \%$ ), pericarditis ( $43 \%$ and $47 \%$ ) (both exudative and adhesive), endocarditis ( $26 \%$ and $33 \%)$, median left ventricular (LV) ejection fraction $(64[59-68] \%$ and $64[61-69] \%)$, LV end-systolic dimension (30[27-32]mm and 29[25-31] mm), LV end-diastolic dimension (48[45-50]mm and 45[42-49]mm), pulmonary artery systolic pressure (25[22-31] $\mathrm{mm} \mathrm{Hg}$ and 23[22-30]mm Hg), LV diastolic disfunction (26\% and $21 \%$ ) and LV systolic dysfunction ( $9 \%$ and $6 \%$ ), LV myocardial hypertrophy (14\% and $21 \%)$ and left atrium dilatation ( $9 \%$ and $21 \%$ ) were found between the "untreated" SLE patients and patients receiving antirheumatic therapy ( $p>0,05$ for all cases). Higher rates of mitral and tricuspid valves prolapse was seen more often in "treatment-naïv" SLE patients: $16(47 \%)$ vs $10(21 \%), p<0,01$

Conclusion: Valvular dysfunction (insufficiency with clinically insignificant regurgitation), pericarditis, endocarditis and LVDD were the most common cardiac TTE abnormalities in SLE patients. Antirheumatic therapy seems not to worsen structural and functional cardiac abnormalities based on TTE findings in SLE patients. Only mitral and tricuspid valves prolapse was seen more often in "treatment-naïv" SLE patients.

Disclosure of Interests: None declared

DOI: 10.1136/annrheumdis-2021-eular.1360 